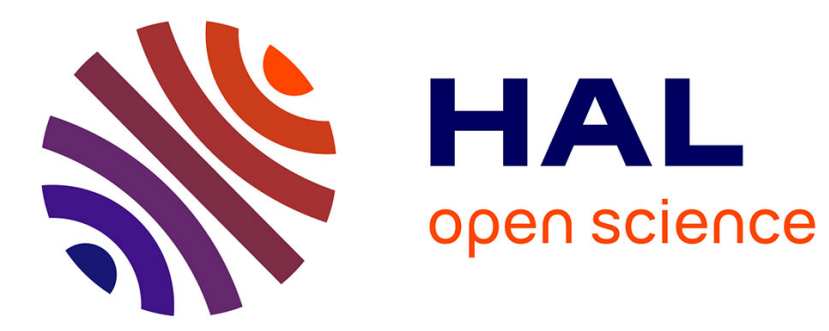

\title{
Metacognitive improvement: Disentangling adaptive training from experimental confounds.
}

Martin Rouy, Vincent de Gardelle, Gabriel Reyes, Jérôme Sackur, Jean Christophe Vergnaud, Elisa Filevich, Nathan Faivre

\section{- To cite this version:}

Martin Rouy, Vincent de Gardelle, Gabriel Reyes, Jérôme Sackur, Jean Christophe Vergnaud, et al.. Metacognitive improvement: Disentangling adaptive training from experimental confounds.. Journal of Experimental Psychology: General, In press, 151 (9), pp.2083-2091. 10.1037/xge0001185 . hal$03581013 \mathrm{v} 2$

\section{HAL Id: hal-03581013 \\ https://hal.science/hal-03581013v2}

Submitted on 9 Jan 2023

HAL is a multi-disciplinary open access archive for the deposit and dissemination of scientific research documents, whether they are published or not. The documents may come from teaching and research institutions in France or abroad, or from public or private research centers.
L'archive ouverte pluridisciplinaire $\mathbf{H A L}$, est destinée au dépôt et à la diffusion de documents scientifiques de niveau recherche, publiés ou non, émanant des établissements d'enseignement et de recherche français ou étrangers, des laboratoires publics ou privés. 


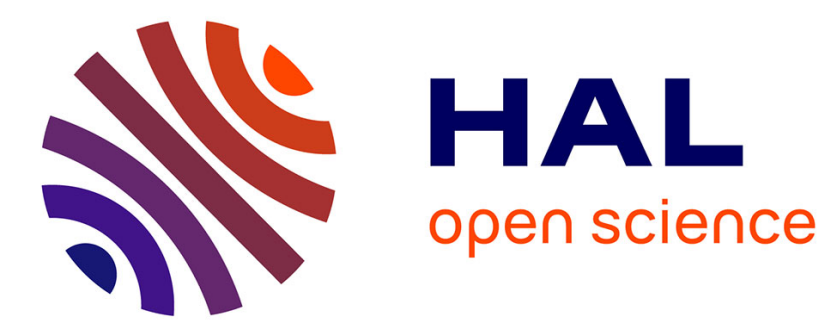

\section{Metacognitive improvement: Disentangling adaptive training from experimental confounds.}

Martin Rouy, Vincent de Gardelle, Gabriel Reyes, Jérôme Sackur, Jean Christophe Vergnaud, Elisa Filevich, Nathan Faivre

\section{- To cite this version:}

Martin Rouy, Vincent de Gardelle, Gabriel Reyes, Jérôme Sackur, Jean Christophe Vergnaud, et al.. Metacognitive improvement: Disentangling adaptive training from experimental confounds.. Journal of Experimental Psychology: General, In press, 151 (9), pp.2083-2091. 10.1037/xge0001185 . hal03581013

\section{HAL Id: hal-03581013 \\ https://hal.archives-ouvertes.fr/hal-03581013}

Submitted on 28 Feb 2022

HAL is a multi-disciplinary open access archive for the deposit and dissemination of scientific research documents, whether they are published or not. The documents may come from teaching and research institutions in France or abroad, or from public or private research centers.
L'archive ouverte pluridisciplinaire HAL, est destinée au dépôt et à la diffusion de documents scientifiques de niveau recherche, publiés ou non, émanant des établissements d'enseignement et de recherche français ou étrangers, des laboratoires publics ou privés. 


\title{
Metacognitive improvement:
}

\section{disentangling adaptive training from experimental confounds}

\author{
Martin Rouy $^{1+}$, Vincent de Gardelle ${ }^{2}$, Gabriel Reyes ${ }^{3}$, Jérôme Sackur ${ }^{4}$, Jean Christophe Vergnaud ${ }^{5}$, \\ Elisa Filevich $^{6,7,8^{*}}$, Nathan Faivre ${ }^{1^{*}}$ \\ 1 Univ. Grenoble Alpes, Univ. Savoie Mont Blanc, CNRS, LPNC, 38000 Grenoble, France \\ 2 Paris School of Economics \& CNRS,Paris, France \\ 3 Universidad Del Desarrollo, Santiago, Chile \\ 4 Laboratoire de Sciences Cognitives et Psycholinguistique, École Normale Supérieure, PSL University, EHESS, CNRS. \\ 5 Centre d'Economie de la Sorbonne, Paris, France \\ 6 Department of Psychology, Humboldt Universität zu Berlin, Unter den Linden 6, 10099 Berlin, Germany \\ 7 Bernstein Center for Computational Neuroscience Berlin, Philippstraße 13 Haus 6, 10115 Berlin, Germany \\ 8 Berlin School of Mind and Brain, Humboldt-Universität zu Berlin, Luisenstraße 56, 10115 Berlin, Germany \\ * equal contribution \\ + Corresponding author: \\ Martin Rouy \\ Laboratoire de Psychologie et Neurocognition \\ CNRS UMR 5105 UGA BSHM \\ 1251 Avenue Centrale \\ 38058 Grenoble Cedex 9 \\ martinrouy03@gmail.com
}

Keywords: cognitive training, metacognition, introspection, confidence

Author Contributions: all authors developed the study concept and contributed to the study design. Modifications in the original code were implemented by EF. Data collection was performed by MR. MR and NF analyzed data. MR and NF drafted the paper; all authors provided critical revisions and approved the final version of the paper for submission. The authors declare no competing interests.

Acknowledgments: NF has received funding from the European Research Council (ERC) under the European Union's Horizon 2020 research and innovation programme (Grant agreement No. 803122). EF was supported by a Freigeist Fellowship from the Volkswagen Foundation (grant number 91620). JS received support from the Agence Nationale de la Recherche, ANR-17-EURE-0017. We thank Steve Fleming for sharing the materials of the original study and commenting on a first version of this manuscript.

Author Note: this work has been presented at the occasion of the 24th annual meeting of the Association for the Scientific Study of Consciousness.

Word count: 3987

Preregistration is publicly available: https://osf.io/gak2t

Data and analysis scripts are publicly available: https://doi.org/10.17605/OSF.IO/RQ967 


\section{Abstract}

Metacognition is defined as the capacity to monitor and control one's own cognitive processes. Recently, Carpenter and colleagues (Carpenter, J., Sherman, M. T., Kievit, R. A., Seth, A. K., Lau, H., \& Fleming, S. M. (2019). Domain-general enhancements of metacognitive ability through adaptive training. Journal of Experimental Psychology: General, 148(1), 51) reported that metacognitive performance can be improved through adaptive training: healthy participants performed a perceptual discrimination task, and subsequently indicated confidence in their response. Metacognitive performance, defined as how much information these confidence judgments contain about the accuracy of perceptual decisions, was found to increase in a group of participants receiving monetary rewards based on their confidence judgments over hundreds of trials and multiple sessions. By contrast, in a control group where only perceptual performance was incentivized, metacognitive performance remained constant across experimental sessions. We identified two possible confounds that may have led to an artificial increase in metacognitive performance, namely the absence of rewards in the initial session and an inconsistency between the reward scheme and the instructions about the confidence scale. We thus conducted a pre-registered conceptual replication where all sessions were rewarded and where instructions were consistent with the reward scheme. Critically, once these two confounds were corrected we found moderate evidence for an absence of metacognitive training. Our data thus suggest that previous claims about metacognitive training are premature, and calls for more research on how to train individuals to monitor their own performance. 


\section{Introduction}

Metacognition is defined as the capacity to monitor and control one's own cognitive processes (Flavell, 1979; Nelson \& Narens, 1994). Metacognitive monitoring is imperfect: Under- or over-estimations regarding the accuracy of one's own judgements are frequent, both in healthy individuals (Shekhar \& Rahnev, 2020, 2021) and in individuals with neurological or psychiatric disorders (Hoven et al., 2019; Rouy et al., 2021). Thus, one outstanding issue is whether one can design training protocols to help individuals improve their abilities to evaluate their own performances.

Recently, Carpenter and colleagues (2019) proposed that metacognitive abilities can be improved through adaptive training. In their study, healthy participants were asked to perform both a memory and a perceptual discrimination task, either with shapes or words stimuli, and subsequently report their confidence in their response. They used a longitudinal protocol in 10 sessions (see Figure 2.A), where the first session (S1, or pre-training session) served as a baseline, followed by eight sessions of training (S2 - S9) on the perceptual task, and finally a post-training session (S10). In the training sessions, participants received feedback and monetary rewards on the basis of their confidence evaluations, after each block of 27 trials: the better the confidence ratings reflected perceptual accuracy in that block, the higher the reward. The pre-training and post-training sessions had no feedback.

Importantly, Carpenter and colleagues reported that metacognitive efficiency, defined as the adequacy between task performance and confidence, increased between pre- and posttraining sessions in the experimental group where participants received monetary rewards on their metacognitive performance, but remained constant in a control group rewarded on their perceptual performance. 
In their paper, Carpenter et al. argued that the increase in metacognitive efficiency that they observed in the post-training session (S10) was mediated by an increase in overall confidence between the pre-training session (S1) and the following session (S2) occurring only in the experimental group. A close inspection of these results reveal that confidence indeed sharply increased from the very beginning of S2, and remained constant afterwards. Likewise, metacognitive performance increased between the pre-training session and S2 but remained constant from S2 onward. This sudden increase in confidence and metacognitive performance suggests that they might have occured due to factors other than training.

We identified two potential confounding factors which we thought could lead to apparent increases in metacognitive efficiency, without involving a real improvement due to training. First, as no reward was offered during the pre-training session, it is possible that the sharp increase in average confidence in S2 reflects a response bias due to the introduction of incentives. Indeed, recent research shows that positive (resp. negative) rewards increased (resp. decreased) confidence irrespective of task performance or metacognitive abilities (Lebreton et al., 2018). Second, the increase in confidence may be driven by differences in the definition of the possible confidence ratings across groups. Indeed, in the pre-training session participants in both the experimental and control groups were instructed to report confidence on a four level scale, defined as 1 = "very low confidence", 2 = "low confidence", $3=$ "high confidence" and 4 = "very high confidence". Importantly no explicit mapping from confidence levels to subjective probabilities was given to participants. In this context, the correct interpretation of the lowest confidence rating is that of a $50 \%$ chance of being correct, i.e. being unsure of the accuracy of their response, and therefore that participants are provided with a half-scale of confidence (Figure 1.A). Yet, from S2 to S9, the experimental 
group (but not the control group) was presented with a full confidence scale, i.e. confidence was mapped onto a probability of a response being correct from 0 to 1 . As a result, confidence ratings 1 and 2 were to be used in case subjects thought they made an error (level 1 would be used when they were certain that they made an error, see Figure 1.A), which rarely occurs in such experimental settings. This full-scale was explained to participants at the beginning of $\mathrm{S} 2$, and implemented in the reward scheme. For instance, according to a fullscale, rating confidence 1 (i.e. "sure incorrect") when incorrect is maximally rewarded (QSR $=1$, see Methods) while rating confidence 1 on a half-scale (i.e. "not sure") is equally rewarded regardless of accuracy (Figure 1.B). Using a full-scale, participants should mostly use the highest ratings, as one can assume that the confident detection of errors is rare in nonspeeded perceptual tasks. Thus, ratings should increase from the first to the second session.

A.

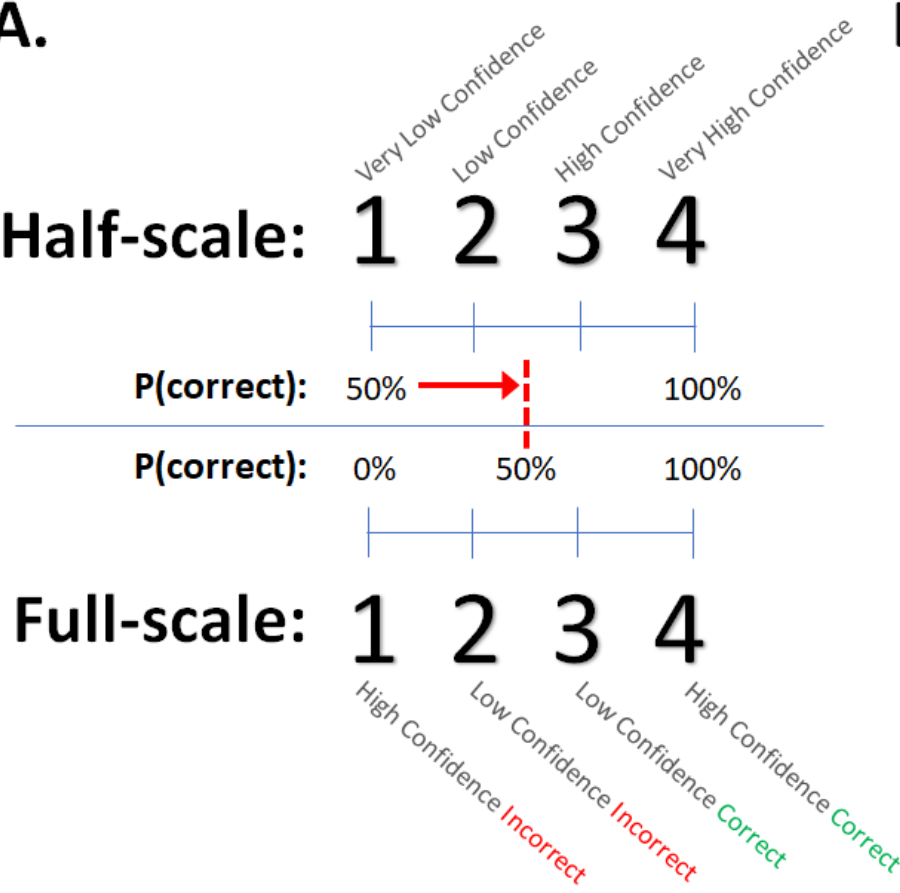

B.

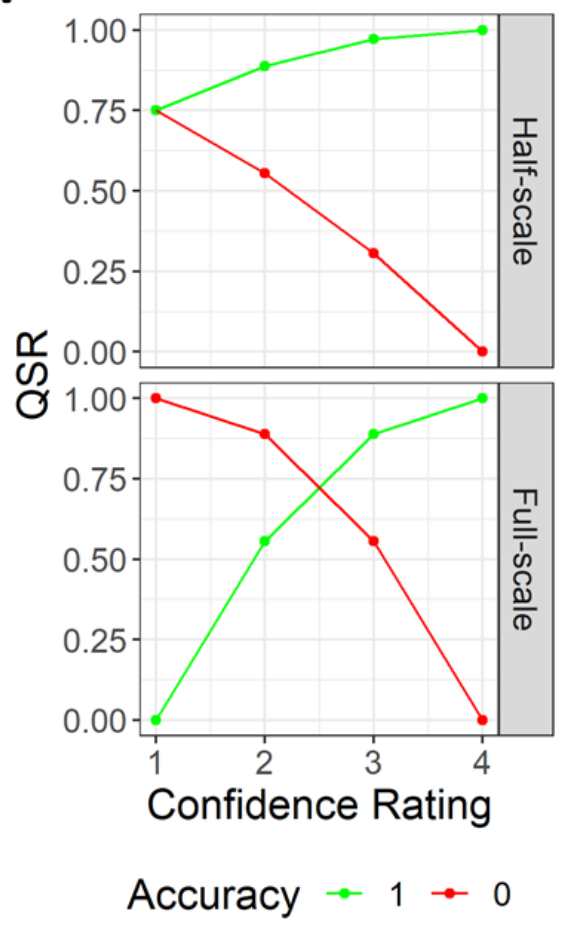

Figure 1. Confidence rating scales. A. Meaning of each confidence rating depending on the type of confidence scale (Half vs. Full), along with the corresponding probability of being 
correct $(\mathrm{P}($ correct $))$. B. Reward schemes depending on the type of confidence scale (Half vs. Full). QSR: Quadratic Scoring rule.

Thus, the introduction of incentives, and the switch from a half-scale to a full-scale may have led to an artificial increase in confidence bias. Importantly, this upward shift in confidence ratings may also be expected to produce an artificial increase in metacognitive efficiency. Indeed, precise confidence criteria might be easier to maintain across two levels on a full scale than four levels on a half-scale. In addition, as suggested in recent works (Shekhar \& Rahnev, 2020, 2021; Xue et al., 2021) criteria for high confidence are noisier than criteria for low confidence and thus a merge of high confidence categories can artificially increase metacognitive efficiency.

In order to assess the contribution of these potential confounds to the observed effects, we reanalyzed the original data, and collected a new dataset attempting to replicate the original findings while controlling for both keeping the incentives and reward scheme constant across sessions (Figure 2.B). Assuming that the original procedure involves genuine metacognitive training, we reasoned that metacognitive efficiency should increase between the first and last session in the experimental group even when issues related to incentives and reward scheme are corrected. Instead, based on a pre-registered sample size of 18 participants, we provide moderate evidence in favor of the null hypothesis according to which adaptive training in the present form does not improve metacognitive ability. 
Methods

Metacognitive performance measurement

To evaluate metacognitive performance, we relied on the M-Ratio measure, derived from the meta-d' framework by Maniscalco and Lau (2012). In signal detection theory, the sensitivity d' quantifies the ability to detect or discriminate a stimulus from the distributions of correct and incorrect responses. Likewise, the metacognitive sensitivity meta-d', quantifies the expected discriminability between two stimuli, if sensory evidence were not degraded between the discrimination decision and confidence rating. Thus, meta-d' refers to the sensory evidence available for metacognition, just as d' is the sensory evidence available for decision-making. It is then possible to quantify how much information was available for the metacognitive task, relative to the information available for the type 1 task, using the ratio meta-d' / d'. This measure, called M-Ratio, is considered as the efficiency of metacognition for each observer.

Reanalysis of original data

We retrieved the original data from the authors and further characterized the evolution of metacognitive performance across sessions with additional mixed-model ANOVAs with Training (Pre-training session vs Post-training session) and Group (Control vs Experimental) as factors. In line with the original mediation analysis, we expected to find a significant increase in metacognitive performance between pre-training session and S2. Furthermore, we compared S2 and S9 to assess the effect of training itself irrespective of the difference in incentives between pre-training session and S2. Statistical analyses were conducted on $\log ($ meta-d'/d'), like in the original study. 
Conceptual replication

Methods and hypotheses were preregistered (https://osf.io/gak2t) prior to data collection.

Modifications from the original study

First, to test the possibility that a difference in terms of incentives between the pre and posttraining sessions might have artificially inflated metacognitive performance, we kept the incentives constant throughout the 10 sessions of the experiment. Accordingly, we refer to the first and last sessions as S1 and S10, instead of the original "pre-training" and "posttraining" sessions, respectively (See Figure 2). In the pre- and post-training sessions, participants in the original study could either start with the memory tasks or the perception tasks. As a consequence of rewarding S1 and S10, participants always started with the perception task. This is to allow for continuity in the explanation of how points were calculated and assigned to participants.

\section{A. Carpenter et al.}

\begin{tabular}{|c|c|c|c|}
\hline \multirow{3}{*}{$\begin{array}{l} \\
\text { Titration } \\
\text { [Perception] }\end{array}$} & $\frac{\text { No feedback }}{432 \text { trials }}$ & $\frac{\text { Training sessions with feedback }}{270 \text { trials/session of perception task }}$ & $\frac{\text { No feedback }}{432 \text { trials }}$ \\
\hline & Pre-training & $\begin{array}{llllllll}\text { S2 } & \text { S3 } & \text { S4 } & \text { S5 } & \text { S6 } & \text { S7 } & \text { S8 } & \text { S9 }\end{array}$ & Post-training \\
\hline & [Memory + Perception] & [Perception] & [Memory + Perception] \\
\hline \multirow{3}{*}{ B. Pre } & Half-scale & Full-scale & Half-scale \\
\hline & \multicolumn{3}{|c|}{ Training sessions with feedback } \\
\hline & 432 trials & 270 trials/session of perception task & 432 trials \\
\hline Titration & S1 & $\begin{array}{llllllll}\text { S2 } & \text { S3 } & \text { S4 } & \text { S5 } & \text { S6 } & \text { S7 } & \text { S8 } & \text { S9 }\end{array}$ & S10 \\
\hline \multirow[t]{2}{*}{ [Perception] } & [Memory + Perception] & [Perception] & [Memory + Perception] \\
\hline & Half-scale & Half-scale & Half-scale \\
\hline
\end{tabular}

Figure 2. Comparison of the original study by Carpenter et al and the present study. A. Original version of the protocol, with pre and post-training sessions providing no feedback, 
and rewards from S2 to S9 mapped onto a full-confidence scale. B. Present version of the protocol, with S1 and S10 providing feedback, and rewards from S2 to S9 mapped onto a half-confidence scale (adapted from Carpenter et al., 2019).

Detailed instructions on how to map confidence to correct and incorrect trials were provided after the titration tasks in S1 but before any task where participants rated confidence. As in the original study, these instructions included a predefined set of demonstration trials and a series of practice trials with trial-wise feedback about whether confidence ratings were correctly assigned to correct or incorrect trials. However, here we made sure that the instructions were consistent with the reward scheme, and that both corresponded to a halfscale.

Second, to assess whether the increase in meta-performance observed in the original study stemmed from an incongruence between instructions regarding the confidence scale and rewards, we provided reward that was consistent with instructions in all sessions: Participants were instructed to report confidence on a four-point scale with $1=$ "very low confidence", 2 = "low confidence", 3 = "high confidence" and 4 = "very high confidence", in all sessions including S1 and S10 (see Figure 2.B). As opposed to the original study, we mapped confidence onto a probability of being correct between 0.5 and 1 , as follows: $P($ correct $)=$ $\frac{\text { conf }+2}{6}$. Subsequently the quadratic scoring rule (QSR) was defined as $1-$ (accuracy $P($ correct $))^{2}$, for each trial (see Figure 1.B).

We also performed minor modifications to the experiment with no consequence on the experimental design: e.g. Carpenter and colleagues ran the initial titration staircase until a fixed number of reversals was reached, or a maximum of 60 trials. We ran the titration staircase for a fixed number of 60 trials. We also fixed a small error in the code shared by Carpenter and colleagues in the memory task resulting in images being presented more than 
once in each block, and other images to never be displayed. All corresponding details are provided in our pre-registration document (https://osf.io/gak2t).

Participants

The sample size was determined according to a pre-registered stopping rule, using an openended sequential Bayes Factor (BF) design. Thus, we tested our effect of interest, namely the interaction between groups (Control vs. Experimental) and sessions (S1 vs. S10) on metacognitive efficiency until moderate evidence toward $\mathrm{H} 1$ or $\mathrm{H} 0$ was reached, i.e. $\mathrm{BF}>5$ or $\mathrm{BF}<0.2$, respectively. As in the original study, we recruited participants through Amazon's MTurk participant marketplace. Sixty-nine participants completed at least the first session. Of these, 11 participants dropped out from the study before the end of the tenth session. Nine participants were excluded for responding incorrectly to screening questions related to the understanding of the tasks, before the beginning of the training (for details, see Carpenter et al., 2019). 19 participants were excluded for technical issues during the first session, leading them to drop at least one experimental condition. Further 11 participants were excluded for either floor $(<55 \%)$ or ceiling $(>95 \%)$ performance in at least one condition/session. Finally, one participant was excluded for reporting the same confidence level on at least $95 \%$ of the trials over three sessions or more. Trials where participants did not respond in time $(>2000 \mathrm{~ms})$ or responded too quickly $(<200 \mathrm{~ms})$ were excluded from further analyses (1.61\% of the trials).

The analyses were conducted on a sample of 18 participants (10 women, mean age: $=40.4$ years, range age $=19-59)$. All participants received monetary compensation in U.S. dollars (range $=\$ 37.6-\$ 41.8)$. An upper bound for sample size was determined using a design analysis with Bayes factors as index of evidence (Schönbrodt \& Wagenmakers, 2018). Data simulations with an expected increase in metacognitive efficiency between S1 and S10 of 
small effect size (Cohen's $d=0.3$ ) revealed that a maximal sample of 100 participants would lead to conclusive evidence under $\mathrm{H} 1$ in $74 \%$ of cases $(\mathrm{BF}>5)$, and under $\mathrm{H} 0$ in $89 \%$ of cases $(\mathrm{BF}<0.2)$. However, the stopping rule criterion was already met when performing the first Bayes Factor sequential analysis after a first group of 18 participants had completed all ten sessions (Figure 5). We recruited participants in the experimental group only (i.e., participants receiving rewards according to metacognitive performance), and compared their data to those of participants in the original control group, who received rewards according to their perceptual performance. As in the original study, bonuses were distributed pseudorandomly to ensure equivalent financial motivation irrespective of performance. The study was approved by the ethics committee from the Paris School of Economics (\#2019 021).

Procedure

Save from the modifications to the code, we used the same HTML/JS/CSS scripts, and therefore the very same stimuli, as in the original study by Carpenter et al. The study ran on a JATOS server (www.jatos.org, Lange et al., 2015).

Statistical analysis

We ran the same analyses as Carpenter and colleagues. We tested for potential changes in metacognitive efficiency $(\log ($ meta-d'/d')) and metacognitive bias (average confidence) using mixed-design ANOVAs in Rstudio version 1.3.1093 (RStudio Team, 2020) using notably the packages tidyverse (Wickham et al., 2019), afex (Singmann and al., 2015), and metaSDT (Craddock, 2018). Bayesian ANOVAs were computed with default prior (Cauchy distribution 
centered on the effect size, with a scaling parameter set to $\frac{\sqrt{2}}{2}$ ) using the BayesFactor package (Morey et al., 2018).

Results

Re-analysis of Carpenter et al. 2019

After confirming the results reported by Carpenter et al, we extended the analyses reported in the original paper in two ways. First, to account for a potential effect of a change in instructions in S2 vs. pre-training, we compared metacognitive efficiency between S2 and the post-training session S10 (instead of between pre- and post-training sessions, as originally reported). Here, we found no significant interaction effect between Group and Training ( $\mathrm{F}(1$, $58)=0.71, \mathrm{p}=0.40, \mathrm{BF}=0.27$ ). When comparing S2 to S9 (i.e. the first and the last of the training sessions), the Group x Training interaction remained non-significant $(F(1,59)=0.49$, $\mathrm{p}=0.49, \mathrm{BF}=0.39)($ Figure 3.A,B). These results suggest that the improvement of metacognitive efficiency occurred not during the extended training part of the protocol, but quite abruptly at the beginning of the training phase.

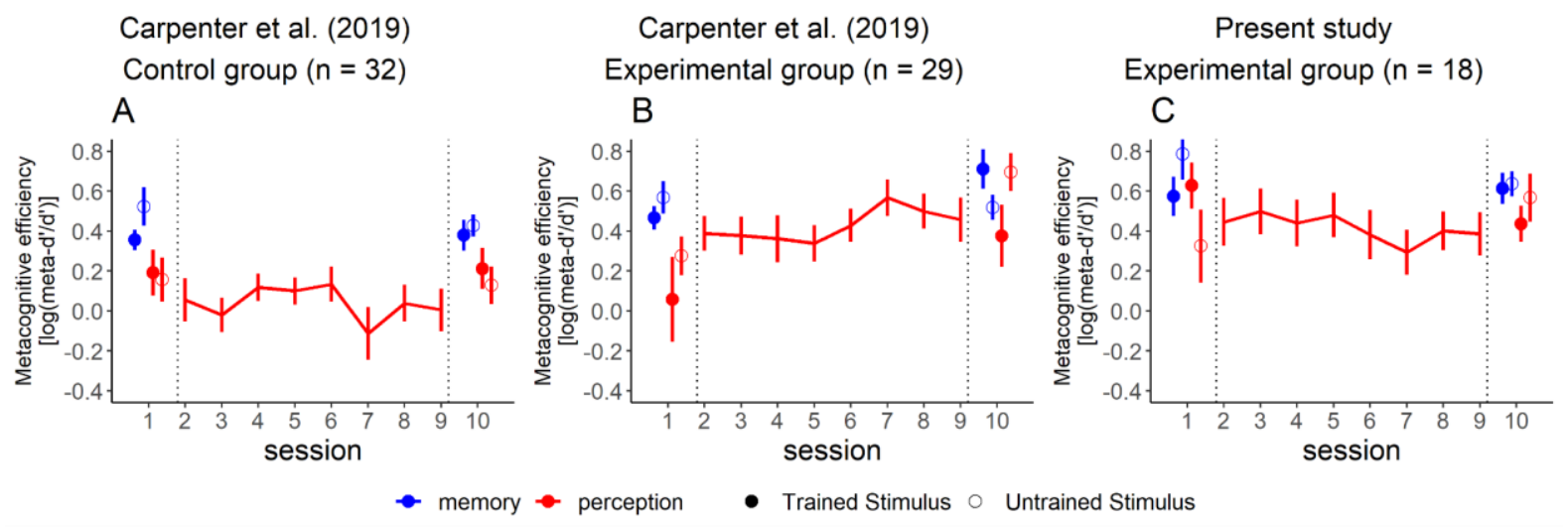

Figure 3. Metacognitive efficiency $(\log ($ meta-d'/d')) over the ten experimental sessions. A, B. Results reproduced from the original data by Carpenter et al, control group and experimental 
group, respectively. C. Results from the present study. Error bars represent standard error of the mean.

Second, we studied the abrupt changes in metacognitive efficiency between the pre-training session and S2. We first found a significant interaction between Group and Training $(\mathrm{F}(1,59)$ $=4.64, \mathrm{p}=0.035)$. Perhaps more strikingly, we found in the original data an abrupt increase in average confidence between the last five trials of the pre-training session and the first five trials of S2 (Figure 4E), in the experimental group only $(\mathrm{F}(1,28)=22.14, \mathrm{p}<.001)$. Together, these results suggest that this increase in metacognitive efficiency could be driven by the changes introduced from S2 to S9, also influencing participants' strategy on the posttraining session $(\mathrm{S} 10)$

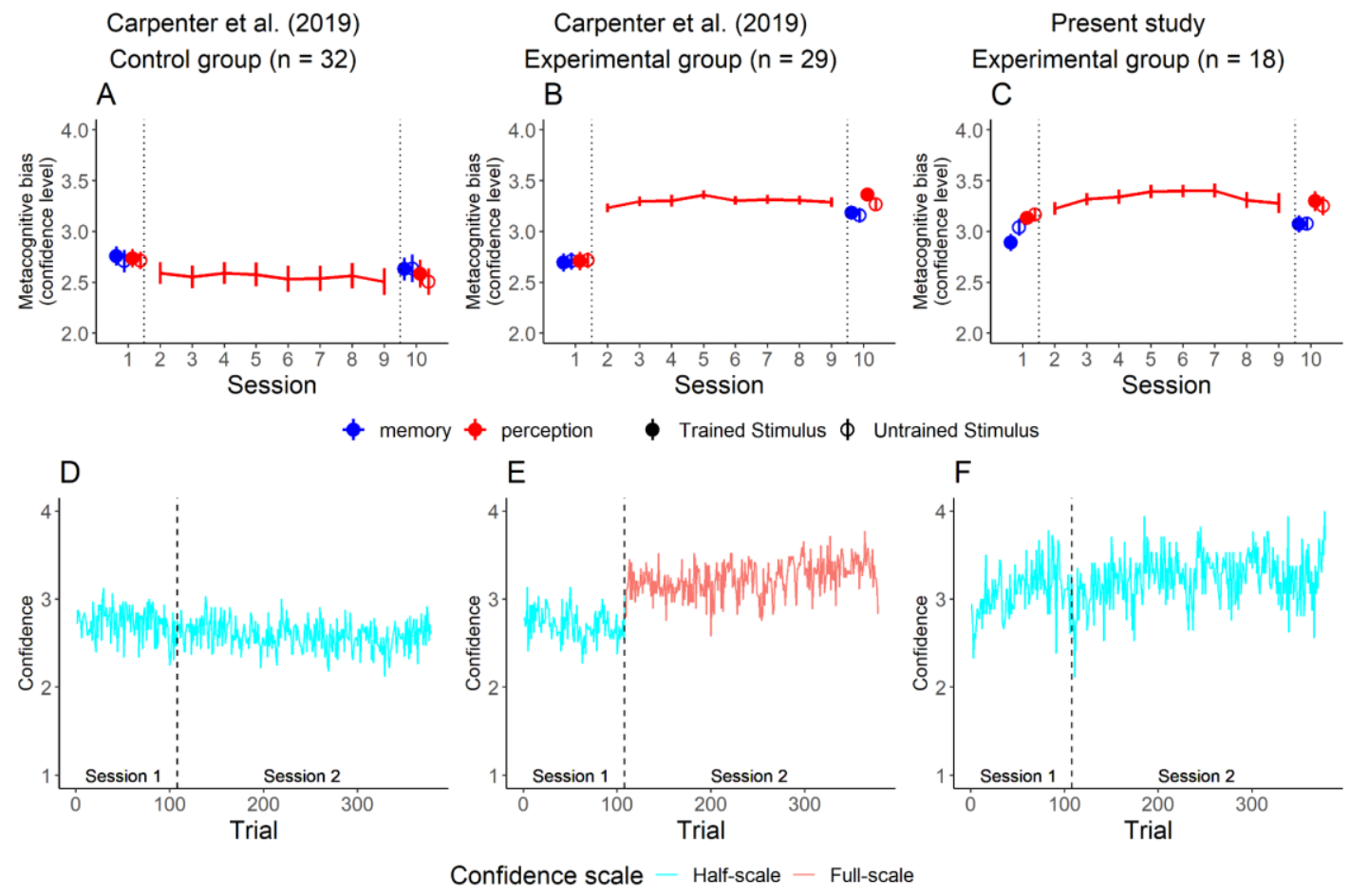

Figure 4. A-C: Metacognitive bias across sessions in the control (A) and experimental groups (B) from Carpenter et al., and in the experimental group from our sample (C). Error bars represent standard error of the mean. D-F: Evolution of average confidence across participants and trials in S1 and S2 in the control (D) and experimental groups (E) from 
Carpenter et al., and in the experimental group from our sample (F). Colors indicate the type of confidence scale in use. Blue: Half-scale, Red: Full-scale.

A pre-registered replication study

Sequential Bayes Factor analysis

Informed by the reanalysis of the original data, we then turned to our conceptual replication study. To assess the efficiency of metacognitive training while accounting for incentives and confidence scale confounding factors, we conducted the same analysis as in the original study comparing metacognitive efficiency $(\log ($ meta-d'/d')) between sessions (S1 and S10) and groups (experimental vs. control).

We had pre-registered recruiting participants until moderate evidence toward $\mathrm{H} 1$ or $\mathrm{H} 0$ was reached.

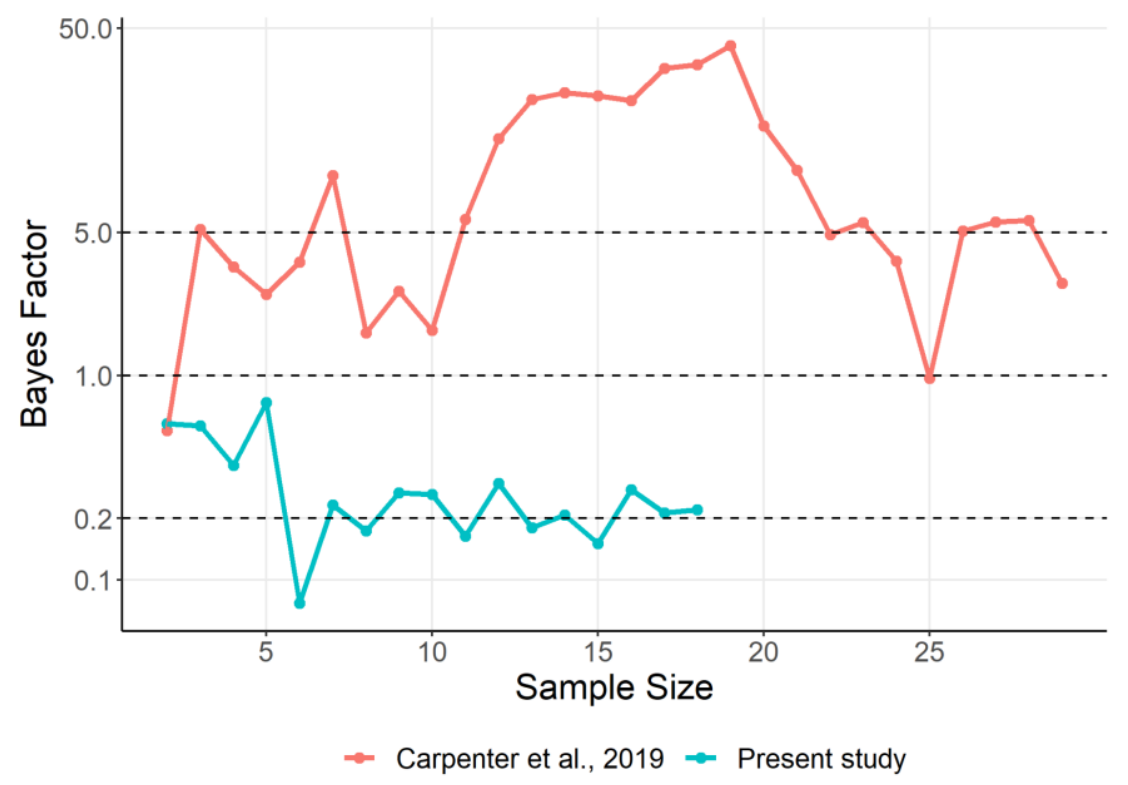

Figure 5. Bayes Factor (BF) sequential analysis of the interaction effect between sessions (S10 and S1) and groups (control vs. experimental) on $\log ($ meta-d'/d)'. The BF assesses whether the effect of interest (interaction Group x Training for metacognitive efficiency) is 
more plausible under $\mathrm{H} 0$ or under $\mathrm{H} 1 \mathrm{BF}>1$ is evidence supporting $\mathrm{H} 1.0<\mathrm{BF}<1$ is evidence supporting H0. The dashed lines mark the ratios where the evidence is five-fold more likely under each hypothesis, which we took as boundaries for moderate evidence. Red curve: Carpenter et al., 2019. Blue curve: Present study.

\section{Metacognitive efficiency}

We compared metacognitive efficiency in S1 and S10 in our new experimental group (Figure 3.C) with those in the control group from Carpenter et al. (2019) (Figure 3.A). Contrary to the original results, the Group $x$ Training interaction was not significant in this analysis $(F(1,45)$ $=0.083, \mathrm{p}=0.93, \mathrm{BF}=0.17)$. Moreover, assessing the linear trend of metacognitive efficiency between S2 and S9 in the three groups, we found no main effect of the training sessions $(\mathrm{F}(7,490)=0.25, \mathrm{p}=0.97, \mathrm{BF}=0.13)$, and no interaction effect between the training sessions and groups (control vs. experimental group in the original study: $F(7,399)=$ $1.61, \mathrm{p}=0.13, \mathrm{BF}=2.50$; control vs. our experimental group: $\mathrm{F}(7,294)=0.90, \mathrm{p}=0.51, \mathrm{BF}$ $=0.24)$. In other words, once we kept the reward scheme constant across all sessions, we found no evidence for metacognitive training in our study. This suggests that previous results might have been confounded by effects of incentives and/or confidence scale, as we detailed in the Introduction.

In their study, Carpenter and colleagues also reported that the peak change in metacognitive efficiency occurred systematically later than the peak change in confidence bias. To assess if a similar pattern was present in our replication group, we conducted an ANOVA with peak session as dependent variable, and outcome (metacognitive efficiency vs. confidence bias) and group (experimental: original vs. replication) as fixed effects. This analysis revealed a main effect of outcome $(F(1,45)=11.37, \mathrm{p}=0.02)$ but no interaction with group $(\mathrm{F}(1,45)=$ $0.01, p=0.98)$, indicating that in both groups the peak change in metacognitive efficiency 
occurred systematically later than the peak change in confidence bias. Since this temporal pattern was also found in our replication group in the absence of global increase in metacognitive efficiency, the extent to which those dynamics are important for metacognitive training remains unclear. Of note, these results are based on a rather small sample size, in compliance with the stopping rule we pre-registered prior to data collection.

Exploring the origin of the Metacognitive bias

Next, we assessed in an exploratory analysis which of the two confounds, incentives or confidence scale, was the main contributor of the confidence increase. This also relates to the question of metacognitive training, as Carpenter and colleagues reported that the increase in metacognitive efficiency was in fact mediated by the increase in metacognitive bias, and as an increase in confidence bias might result in an increase in metacognitive efficiency (Shekhar and Rahnev, 2021).

If this abrupt increase in confidence ratings was due to the introduction of incentives at S2, we would expect the same average confidence in our experimental group (Figure 4.C) and from S2 to S9 in the original experimental group, as these conditions are similar in terms of rewards. We would also expect these two conditions to show higher levels of confidence than the control group. This is what we found in the data. When comparing average confidence in S2-S9 between the 3 groups (control vs. original experimental vs. replication) with an ANOVA, we found a main effect of $\operatorname{Group}(\mathrm{F}(2,72)=24.61, \mathrm{p}<.001)$, driven by significantly higher levels of confidence both in our replication group $(\mathrm{t}(72)=-5.05, \mathrm{p}<$ $.001)$ and in the original experimental group $(t(72)=-6.43, \mathrm{p}<.001)$, compared to the control group, with no difference between the experimental group and the replication group $(\mathrm{t}(72)=$ $0.10, \mathrm{p}=0.995, \mathrm{BF}=0.46)$. However, we are cautious in interpreting confidence biases that might not be comparable between groups and studies. 
One other possibility is that this abrupt increase in average confidence was due to a shift in the type of confidence scale (i.e. half-scale in the pre-training session, and full-scale from S2 to S9, see Figure 2.A). If this were true, then we would expect the average confidence in our replication group (which used a half-confidence scale) to be lower than the level of confidence obtained from S2 to S9 in the original experimental group. As just mentioned, however, these two conditions were not different in terms of average confidence.

Furthermore, because the increased levels of confidence described above are not accompanied by an increase in first-order performance (as assessed through difficulty levels across the three groups, $\mathrm{F}(2,72)=0.17, \mathrm{p}=0.84, \mathrm{BF}=0.18)$ it is unlikely that the metacognitive bias can simply be explained by a generic motivation effect.

Altogether, these analyses thus suggest that the presence of incentives might be the main reason for the increase in confidence ratings, which in turn would have led to an increase of metacognitive efficiency, as recently proposed (Shekhar and Rahnev, 2021). Nonetheless, as our analyses relied on comparing confidence biases between studies in relatively small samples, these conclusions on the specific mechanism at stake should be taken with caution.

\section{Discussion}

In the present work, we aimed at re-assessing the effectiveness of a protocol designed by Carpenter and colleagues (2019) to improve metacognitive abilities. We noticed that the increase in metacognitive efficiency found by Carpenter and colleagues might be unspecific, due to an artificial increase in confidence bias, triggered by two confounding factors: In the original study, reward was not held constant throughout all sessions, so that participants might have been more incentivized to perform the task not only during rewarded sessions (S2-S9), but also in the post-training session (S10), as a spillover effect. Also, the instructions 
provided to the participants in the experimental group were not congruent with the reward scheme, encouraging them to use high confidence ratings (i.e. ratings 3 and 4) from S2 onward but not in the pre-training session. To evaluate our claim that the original results may be due to confounding factors, we performed additional analyses on the original data set. First, when restricting the analysis to training sessions only (i.e., S2 to S9, instead of pretraining and post-training sessions), thus controlling for incentives, we found no evidence for an improvement in metacognitive performance in the experimental group. By contrast, this increase was already significant between S1 and S2. This sharp increase in metacognitive performance was accompanied by an abrupt increase in average confidence between the last trials of the pre-training session and the first trials of S2. In our view, the fact these behavioral changes occurred rapidly in time at the very beginning of the experimental procedure casts doubts on the possibility that they arose due to a genuine improvement in metacognitive performance. Instead, we suspect that they may have been due to either, or both, of the two possible experimental confounds mentioned above.

To further assess the validity of this training procedure, we conducted a conceptual replication controlling for both incentives and confidence-related factors by, first, providing rewards in all sessions (i.e. including S1 and S10) and, second, rewarding the experimental group on the basis of a half-confidence scale, in line with the instructions received by participants (and instead of a full-scale as in the original study). We reasoned that, if the training method was effective in improving metacognition, estimates of metacognitive efficiency should increase between S1 and S10 in the experimental group, even when issues related to incentives and confidence scale were corrected. Instead, we obtained moderate evidence in favor of $\mathrm{H} 0$ (following a pre-registered open-ended sequential Bayes factor analysis), indicating that no increase in metacognitive efficiency occurred. Thus, we suggest 
that the increase in metacognitive efficiency reported by Carpenter et al (2019) resulted from a global change in the use of the confidence scale, possibly due to incentives or instructions regarding the confidence scale, rather than from an improved sensitivity to trial-wise fluctuations in the quality of the decision. While such a global adjustment of confidence ratings might be adaptive and useful (for example when communicating confidence in order to reach joint decisions), it is important to distinguish this effect from a genuine improvement of metacognitive monitoring, conceptually and empirically. Of note, post-hoc analyses revealed that metacognitive efficiency in S1 was higher in the replication compared to the original experimental group with marginal significance $(\mathrm{p}=0.11)$, probably due to the fact that $\mathrm{S} 1$ in our replication group was rewarded, pushing participants to perform better. Yet, it might be that metacognitive efficiency in the replication group reached a ceiling early in the procedure, leaving little room for improvement even if training were in fact possible under this new protocol.

In recent years, the field of metacognition has seen a dramatic increase in popularity, in part due to the development of new statistical tools that allow quantifying metacognitive performance independently from typical confounds such as first-order performance (Fleming \& Lau, 2014; Galvin et al., 2003; Maniscalco \& Lau, 2012). Moreover, metacognitive deficits are prevalent in several psychiatric and neurological disorders, with severe consequences in terms of medical observance and quality of life (Hasson-Ohayon et al., 2015; Lysaker et al., 2015). This is why developing robust, efficient, and cost-effective remediation procedures to improve metacognitive performance is important. Several studies already provided evidence suggesting that monitoring abilities can be trained: A two-week meditation training was found to enhance metacognitive accuracy in the memory domain (Baird et al., 2014), and knowledge about cognitive biases is held to reduce delusions and 
positive symptoms in schizophrenia (for a review, see Eichner \& Berna, 2016). More recently, preliminary results from a virtual-reality assisted training consisting in frequently questioning the reality of wakeful experiences augmented the rate of lucid dreaming experiences (Gott et al., 2021). Despite pioneering experiments showing promising results (Adams \& Adams, 1959; Sharp et al., 1988), to our knowledge, no recent remediation procedure based on feedback has been successful in improving the quality of confidence ratings (for a recent attempt based on single-trial feedback, see Haddara \& Rahnev (2019, 2020)).

Future attempts to improve the quality of confidence ratings may be informed by recent findings regarding the definition of metacognitive noise (Shekhar \& Rahnev, 2020, 2021; Xue et al., 2021), as a way to provide more information to participants regarding the qualitative nature of their metacognitive deficits. They could also rely on elicitation methods that encourage participants to report optimal confidence estimates, such as measuring participants' willingness to trade a gamble based on the accuracy of their response against a lottery with known probabilities (Dienes and Seth, 2010; Massoni et al., 2014). Another way of refining confidence ratings may be to provide participants with feedback regarding the temporal dynamics with which first-order decisions are made. Indeed, becoming aware of how the decision-making process unfolds in time may help to better judge the accuracy of a given decision. Practically, this could simply consist in presenting participants with feedback about their own response times for correct and incorrect responses, or more ambitiously with parameter estimates from mouse-tracking (Faivre et al., 2019; Dotan 2019) or post-decisional evidence accumulation models (Pleskac \& Busemeyer 2010; Pereira et al., 2019; 2021). Other strategies may consist in training participants to better detect their attentional lapses (Baird et al., 2014; Recht et al., 2021), or to regulate brain networks associated with over or 
underconfidence (Cortese et al., 2016). Given the complexity of this endeavor, and the societal and clinical issues at stake, effective metacognitive training will probably require collective efforts rather than individual initiatives (Rahnev et al., 2021). In that regard, we highlight the openness from the authors of the original study, who publicly shared their valuable code and data and discussed these results openly with us, as those are the first necessary steps towards collective research on metacognition.

\section{Context of the Research}

We were interested in the possibility to train metacognitive abilities in the broader context of our research on schizophrenia. A rich clinical literature suggests the existence of metacognitive deficits in individuals with schizophrenia, and efforts had already been made to alleviate symptoms and improve quality of life through metacognitive training. Existing metacognitive training procedures rely on explicit and high-level strategies, notably by encouraging patients to bring unnoticed beliefs and cognitive biases to awareness. As a complementary intervention, we were enthusiastic about the metacognitive training proposed by Carpenter and colleagues, which targeted lower-level mechanisms involved in learning how to properly estimate confidence on a trial-to-trial basis. If successful in healthy participants, we were hoping to adapt this procedure to clinical settings. 


\section{References}

Adams, P. A., \& Adams, J. K. (1958). Training in confidence-judgments. The American Journal of Psychology, 71(4), 747-751.

Baird, B., Mrazek, M. D., Phillips, D. T., \& Schooler, J. W. (2014). Domain-specific enhancement of metacognitive ability following meditation training. Journal of Experimental Psychology: General, 143(5), 1972-1979. https://doi.org/10.1037/a0036882

Carpenter, J., Sherman, M. T., Kievit, R. A., Seth, A. K., Lau, H., \& Fleming, S. M. (2019). Domain-general enhancements of metacognitive ability through adaptive training. Journal of Experimental Psychology: General, 148(1), 51-64. https://doi.org/10.1037/xge0000505

Cortese, A., Amano, K., Koizumi, A., Kawato, M., \& Lau, H. (2016). Multivoxel neurofeedback selectively modulates confidence without changing perceptual performance. Nature communications, 7(1), 1-18.

Craddock, M. (2018). metaSDT: Calculate Type 1 and Type 2 Signal Detection Measures. R package version 0.5 .0 .

Dienes, Z., \& Seth, A. (2010). Gambling on the unconscious: A comparison of wagering and confidence ratings as measures of awareness in an artificial grammar task. Consciousness and cognition, 19(2), 674-681.

Dotan, D., Pinheiro-Chagas, P., Al Roumi, F., \& Dehaene, S. (2019). Track it to crack it: dissecting processing stages with finger tracking. Trends in Cognitive Sciences, 23(12), 1058-1070.

Eichner, C., \& Berna, F. (2016). Acceptance and Efficacy of Metacognitive Training (MCT) on Positive Symptoms and Delusions in Patients With Schizophrenia: A Metaanalysis Taking Into Account Important Moderators. Schizophrenia Bulletin, 42(4), 
952-962. https://doi.org/10.1093/schbul/sbv225

Faivre, N., Roger, M., Pereira, M., de Gardelle, V., Vergnaud, J. C., Passerieux, C., \& Roux, P. (2021). Confidence in visual motion discrimination is preserved in individuals with schizophrenia. Journal of Psychiatry \& Neuroscience: JPN, 46(1), E65.

Faivre, N., Filevich, E., Martin, R., de Gardelle, V., Vergnaud, J., \& Reyes, G. (2020, March 23). Replication: Domain-general Enhancements of Metacognitive Ability Through Adaptive Training. https://doi.org/10.17605/OSF.IO/GAK2T

Flavell, J. H. (1979). Metacognition and cognitive monitoring: A new area of cognitivedevelopmental inquiry. American psychologist, 34(10), 906.

Fleming, S. M., \& Lau, H. C. (2014). How to measure metacognition. Frontiers in Human Neuroscience, 8. https://doi.org/10.3389/fnhum.2014.00443

Fox J, Weisberg S (2019). An R Companion to Applied Regression, Third edition. Sage, Thousand Oaks CA. https://socialsciences.mcmaster.ca/jfox/Books/Companion/. Galvin, S. J., Podd, J. V., Drga, V., \& Whitmore, J. (2003). Type 2 tasks in the theory of signal detectability: Discrimination between correct and incorrect decisions. Psychonomic Bulletin \& Review, 10(4), 843-876.

https://doi.org/10.3758/BF03196546

Gott, J., Bovy, L., Peters, E., Tzioridou, S., Meo, S., Demirel, Ç., Esfahani, M. J., Oliveira, P. R., Houweling, T., Orticoni, A., Rademaker, A., Booltink, D., Varatheeswaran, R., van Hooijdonk, C., Chaabou, M., Mangiaruga, A., van den Berge, E., Weber, F. D., Ritter, S., \& Dresler, M. (2021). Virtual reality training of lucid dreaming. Philosophical Transactions of the Royal Society B: Biological Sciences, 376(1817), 20190697. https://doi.org/10.1098/rstb.2019.0697

Guggenmos, M. (2021). Validity and reliability of metacognitive performance measures. 
Haddara, N., \& Rahnev, D. (2019). Trial-by-trial feedback does not improve performance or metacognition in a large-sample perceptual task. Journal of Vision, 19(10), 27-27.

Haddara, N., \& Rahnev, D. (2020). The impact of feedback on perceptual decision making and metacognition: Reduction in bias but no change in sensitivity.

Hasson-Ohayon, I., Avidan-Msika, M., Mashiach-Eizenberg, M., Kravetz, S., Rozencwaig, S., Shalev, H., \& Lysaker, P. H. (2015). Metacognitive and social cognition approaches to understanding the impact of schizophrenia on social quality of life. Schizophrenia Research, 161(2-3), 386-391.

Hoven, M., Lebreton, M., Engelmann, J. B., Denys, D., Luigjes, J., \& van Holst, R. J. (2019). Abnormalities of confidence in psychiatry: An overview and future

perspectives. Translational Psychiatry, 9(1), 268. https://doi.org/10.1038/s41398-0190602-7

Lange, K., Kühn, S., \& Filevich, E. (2015). "Just Another Tool for Online Studies" (JATOS): An Easy Solution for Setup and Management of Web Servers Supporting Online Studies. PLOS ONE, 10(6), e0130834.

https://doi.org/10.1371/journal.pone.0130834

Lebreton, M., Langdon, S., Slieker, M. J., Nooitgedacht, J. S., Goudriaan, A. E., Denys, D., ... \& Luigjes, J. (2018). Two sides of the same coin: Monetary incentives concurrently improve and bias confidence judgments. Science Advances, 4(5), eaaq0668.

Lysaker, P. H., Vohs, J., Minor, K. S., Irarrázaval, L., Leonhardt, B., Hamm, J. A., ... \& Dimaggio, G. (2015). Metacognitive deficits in schizophrenia: presence and associations with psychosocial outcomes. The Journal of nervous and mental disease, 203(7), 530-536.

Maniscalco, B., \& Lau, H. (2012). A signal detection theoretic approach for 
estimating metacognitive sensitivity from confidence ratings. Consciousness and Cognition, 21(1), 422-430. https://doi.org/10.1016/j.concog.2011.09.021

Massoni, S., Gajdos, T., \& Vergnaud, J. C. (2014). Confidence measurement in the light of signal detection theory. Frontiers in psychology, 5, 1455.

Morey, R. D., Rouder, J. N., Jamil, T., \& Urbanek, S. (2018). BayesFactor: Computation of Bayes Factors for common designs. R package version 0.9. 12-4.2.

Moritz, S., \& Woodward, T. S. (2007). Metacognitive training in schizophrenia: From basic research to knowledge translation and intervention. Current Opinion in Psychiatry, 20(6), 619-625. https://doi.org/10.1097/YCO.0b013e3282f0b8ed Pereira, M.*, Faivre, N.*, Iturrate, I.*, Wirthlin, M., Serafini, L., Martin, S., Desvachez, A., Blanke, O., Van De Ville, D., and Millan, J. (2020). Disentangling the origins of confidence in speeded perceptual judgments through multimodal imaging. PNAS, doi: 10.1073/pnas.1918335117

Pereira, M., Megevand, P., Tan, M. X., Chang, W., Wang, S., Rezai, A., ... \& Faivre, N. (2021). Evidence accumulation relates to perceptual consciousness and monitoring. Nature communications, 12(1), 1-11.

Pleskac, T. J., \& Busemeyer, J. R. (2010). Two-stage dynamic signal detection: a theory of choice, decision time, and confidence. Psychological review, 117(3), 864.

Nelson, T. O., \& Narens, L. (1994). Why investigate metacognition. Metacognition: Knowing about knowing, 13, 1-25.

RStudio Team (2020). RStudio: Integrated Development. Environment for R. RStudio, PBC, Boston, MA URL. http://www.rstudio.com/.

Recht, S., de Gardelle, V., \& Mamassian, P. (2021). Metacognitive blindness in temporal selection during the deployment of spatial attention. Cognition, 216, 104864. 
Rouy, M., Saliou, P., Nalborczyk, L., Pereira, M., Roux, P., and Faivre, N. (2021) Systematic review and meta-analysis of metacognitive abilities in individuals with schizophrenia spectrum disorders. Neuroscience and Biobehavioral Reviews, doi: 10.1016/j.neubiorev.2021.03.017

Rouy, M., de Gardelle, V., Vergnaud, J.-C., Reyes, G., Filevich, E., \& Faivre, N. (2021, November 19). Replication: Domain-general Enhancements of Metacognitive Ability Through Adaptive Training. https://doi.org/10.17605/OSF.IO/RQ967

Schönbrodt, F. D., \& Wagenmakers, E.-J. (2018). Bayes factor design analysis:

Planning for compelling evidence. Psychonomic Bulletin \& Review, 25(1), 128-142. https://doi.org/10.3758/s13423-017-1230-y

Sharp, G. L., Cutler, B. L., \& Penrod, S. D. (1988). Performance feedback improves the resolution of confidence judgments. Organizational Behavior and Human Decision Processes, 42(3), 271-283.

Shekhar, M., \& Rahnev, D. (2020). Sources of Metacognitive Inefficiency. Trends in Cognitive Sciences.

Shekhar, M., \& Rahnev, D. (2021). The nature of metacognitive inefficiency in perceptual decision making. Psychological Review, 128(1), 45.

Singmann, H., Bolker, B., Westfall, J., Aust, F., \& Ben-Shachar, M. S. (2015). afex: Analysis of factorial experiments. R package version $0.13-145$.

Wickham, H., et al., (2019). Welcome to the Tidyverse. Journal of Open Source Software, 4(43), 1686. https://doi.org/10.21105/joss.01686

Xue, K., Shekhar, M., \& Rahnev, D. The nature of metacognitive noise confounds metacognitive sensitivity and metacognitive bias. 
